REVIEW

\title{
Cubital tunnel syndrome
}

\section{Steven Cutts}

Postgrad Med J 2007;83:28-31. doi: 10.1136/pgmi.2006.047456

Cubital tunnel syndrome is the second most common peripheral nerve entrapment syndrome in the human body. It is the cause of considerable pain and disability for patients. When appropriately diagnosed, this condition may be treated by both conservative and operative means. In this review, the current thinking on this important and common condition is discussed The recent literature on cubital tunnel syndrome was reviewed, and key papers on upper limb and hand surgery were discussed with colleagues.

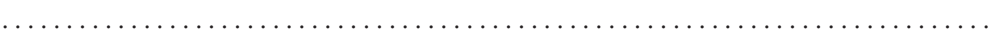

Correspondence to: S Cutts, 3 Watchets Green, Lyppard, Habington, Worcester WR4 ORT, UK; stevenfrcs@hotmail.com

Received 7 March 2006 Accepted 28 April 2006

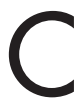
ubital tunnel syndrome is the second most common peripheral nerve entrapment neuropathy in the upper limb. It represents a source of considerable discomfort and disability for the patient, and in extreme cases may progress to loss of function of the hand. Cubital tunnel syndrome remains an often misdiagnosed condition.

In this article, I consider the aetiology of the syndrome, its clinical presentation and current concepts regarding treatment.

\section{THE ANATOMY OF THE ULNAR NERVE AND THE CUBITAL TUNNEL}

The cubital tunnel is formed by the cubital tunnel retinaculum which straddles a gap of about $4 \mathrm{~mm}$ between the medial epicondyle and the olecranon. ${ }^{1}$ In turn, the floor of the tunnel is formed by the capsule and the posterior band of the medial collateral ligament of the elbow joint. It contains several structures, the most important of which is the ulnar nerve.

The ulnar nerve is the terminal branch of the medial cord of the brachial plexus, and contains fibres from the $\mathrm{C} 8$ and $\mathrm{Tl}$ spinal nerve roots. It descends the arm just anterior to the medial intermuscular septum and later pierces this septum in the final third of its length. Progressing underneath the septum and adjacent to the triceps muscle, it traverses the cubital tunnel to enter the forearm where it passes between the two heads of the flexor carpi ulnaris muscle.

This anatomical arrangement has two implications for the nerve. Firstly, the ulnar follows a relatively constrained path, and secondly, it lies some distance from the axis of rotation of the elbow joint. Movement of the elbow therefore requires the nerve to both stretch and slide through the cubital tunnel. Sliding has the greatest role in this process, although the nerve itself can stretch by up to $5 \mathrm{~mm}$.

The unusual anatomy of the cubital tunnel and the well-recognised increase in intraneural pressure associated with elbow flexion are believed to be key issues in the pathogenesis of cubital tunnel syndrome. In addition, the shape of the tunnel changes from an oval to an ellipse with elbow flexion. This manoeuvre also narrows the canal by $55 \%$. Elbow flexion, wrist extension and shoulder abduction increases intraneural pressure by six times.

There are five principal locations where the ulnar nerve may be compressed around the elbow:

(1) Arcade of Struthers

(2) medial intermuscular septum

(3) medial epicondyle

(4) cubital tunnel

(5) deep flexor aponeurosis.

Of these, the cubital tunnel is by far the most common.

It has long been recognised that the substance of the cubital tunnel retinaculum can vary dramatically between individuals. In 1991, O'Driscoll et al published the results of an extensive (27 cases) cadaveric study in which he attempted to divide these variations into four types. In a small proportion of patients, the retinacular tissue was found to be completely absent (type 0 ). In othersas was already understood-it is a muscular structure known as the anconeus epitrochlearis (O'Driscoll type II). O'Driscoll considered a fibrous retinaculum to be more usual, and in this situation, it is traditionally referred to as the arcuate ligament or Osborne's band. Phylogenetically, O'Driscoll considered the arcuate ligament to represent a remnant of the anconeus epitrochlearis muscle.

This detailed study also noted that the tightness of the fibrous retinaculum varied with the position of the elbow. Most commonly (type Ia), it was tense in full flexion and lax in extension, with a few cases being tense at $90-120^{\circ}$ of flexion (type Ib). O'Driscoll went on to speculate that these subtle variations in anatomy might explain why some patients seem to be more predisposed to cubital tunnel syndrome than others. For example, an absent ligament may predispose to subluxation of the nerve.

The Arcade of Struthers is another, variable structure that is a rare cause of primary cubital tunnel syndrome. In a cadaveric study of 60 limbs, Siqueria and Mortins ${ }^{2}$ reported only 8 (13.5\%) limbs where this musculotendinous structure was discernable, 3-10 $\mathrm{cm}$ above the medial epicondyle. When present, it did not seem to compress the ulnar nerve.

However, Siqueria also acknowledged that the Arcade of Struthers functions as a likely site for 
Box 1: Anatomical sites for ulnar nerve compression

(1) C8 radiculopathy

(2) Thoracic outlet syndrome

(3) The cubital tunnel itself

(4) Compression within Guyon's canal

(5) Two or more of the above-that is, double crush syndrome

"secondary compression". After transposition of the ulnar nerve, it is common for the mobilised nerve to come under tension on the Arcade of Struthers or medial intermuscular septum, causing secondary impingement.

\section{WHO GETS CUBITAL TUNNEL SYNDROME?}

As with all nerve disorders, patients with diabetes mellitus are at increased risk of ulnar nerve symptoms.

Cubital tunnel syndrome is also more common in patients whose work involves protracted periods of elbow flexion (such as holding telephones). In particular, flexion with the elbow pressed against a hard surface increases the risk of cubital tunnel syndrome, at least partly, because of the increase in the intraneural pressure in this position. People who have had a direct blow against the ulnar nerve are also at risk, as are those with marked varus or valgus deformity at the elbow. ${ }^{3}$

Descatha et al, ${ }^{4}$ working in Scandinavia, found that the major risk factors for cubital tunnel syndrome were obesity and holding a tool in a constant position, performing a repetitive task. The existence of other upper limb work-related musculoskeletal disorders was also found to be a risk factor. Such disorders include medial epicondylitis (golfers elbow) and other upper limb entrapment syndromes such as cervicobrachial neuralgia, carpal tunnel syndrome and radial tunnel syndrome.

Kakosy ${ }^{5}$ studied Hungarian workers operating vibrating tools and found an increased rate of upper limb neurology, including cubital tunnel syndrome in $42.5 \%$ of 167 patients.

The American and Japanese literature places a heavy emphasis on the susceptibility of baseball throwers to cubital tunnel syndrome. Ulnar nerve symptoms during that part of the throwing cycle that involves extreme flexion (late cocking, early acceleration) is strongly suggestive of cubital tunnel syndrome. ${ }^{6}$

Seror and Nathan ${ }^{7}$ investigated 882 French and 818 American patients who had upper limb electrical tests. In both countries, the risk of an abnormal electrical test was 2 to 1 for wrist versus elbow. However, a median nerve with an abnormal electrical test was twice as likely to be symptomatic as an ulnar nerve with abnormal electrical tests, with the result that the

\section{Box 2: Risks of surgical procedure}

- Complications of ulnar nerve release

- Persistent dysaesthesia

- Reflex sympathetic dystrophy

- Haematoma

- Infection

- Neuroma of the medial brachial and medial antebrachial cutaneous nerves

- Persistent sensory deficit

- Persistent weakness

- Medial epicondylectomy

- Risk of damage to medial collateral ligaments ratio of median to ulnar clinical problems was 4 to 1 . These results imply that, in some patients with carpal tunnel syndrome who continue to have symptoms after surgery, the underlying problem may be an undiagnosed cubital tunnel syndrome.

\section{MECHANISMS FOR PERIPHERAL NERVE DAMAGE}

Compression, traction and friction have been implicated in cubital tunnel syndrome.

Compression is usually regarded as the principal mechanism of nerve damage in peripheral neuropathy. Damage may occur either by direct mechanical compression or by compression of the intrinsic blood supply to the nerve, which in turn causes local ischaemia. Mechanical compression forces of $\geqslant 30 \mathrm{~mm} \mathrm{Hg}$ retard blood flow. ${ }^{8}$ Similarly, compression has been shown to interfere with axonal transport pathways.

Larger fibres containing more myelin are more susceptible to compression than smaller non-myelinated fibres. ${ }^{9}$ Compression is most effective at the edge of the compression area in the socalled "edge effect".

Pre-existing subclinical mechanical compression of the nerve at a different location (box 1) may increase the susceptibility of the same nerve to compression at a second, more distal, site (the so-called "double crush" phenomenon. ${ }^{10}{ }^{11}$

Previous injuries to the nerve may tether it to the walls of the tunnel, prevent normal sliding and expose it to traction injuries. Similarly, a tight tunnel may predispose the nerve to friction and compression.

\section{DIABETES MELLITUS}

Diabetes makes a nerve more vulnerable to compression. This may occur secondary to a microvascular injury in the nerve causing local ischaemia or by interfering with the innate metabolism of the nerve. There is evidence of damage to axonal transport in the nerve. Diabetes may increase the risk of damage in a manner similar to mechanical double crush. ${ }^{12}$

\section{SYMPTOMS AND SIGNS OF ULNAR NERVE IMPINGEMENT}

Patients with ulnar nerve compression at any level have altered sensation in the little and ring fingers. Indeed, in most patients, sensory loss is the first symptom to be reported. As the condition progresses, they may also notice clumsiness in the hand, as the ulnar nerve is the principal motor supply to the intrinsic muscles of the hand. In well-established cases, there may be marked wasting of the small muscles of the hand and the ulnar-sided muscles of the forearm.

The examining doctor may look for Froment's sign, overt clawing of the ulnar-innervated digits (usually the little and ring fingers) and abduction of the little fingers (Wartenberg's sign).

Inspection of the elbow in extension may show a valgus deformity, possibly secondary to a previous fracture around the elbow. Malunion after supracondylar fracture of the humerus can result in an adult cubitus valgus deformity, which in turn predisposes to a tardy ulnar nerve palsy. ${ }^{13}$

In a non-traumatic case of cubital tunnel syndrome, the most likely cause is ulnar nerve entrapment, but the nerve can be compressed at any position along its length (box 1 ).

In the unusual case of compression in Guyon's canal, sensation is preserved over the dorsum of the hand. Here sensation is from the dorsal cutaneous branch of the ulnar nerve that comes off proximal to Guyon's canal, and therefore remains intact.

Tinel's sign should be positive over the cubital tunnel itself, although some surgeons find it easier to elicit Tinel's sign over the medial side of the humerus. 


\section{Box 3: McGowan Score}

Grading system for ulnar nerve neuropathy

(1) Mild occasional paraesthesia, positive Tinel's sign, subjective weakness

(2) Moderate paraesthesia, objective weakness, positive Tinel's sign

(3) Severe constant paraesthesia, weakness, overt muscle wasting

The elbow flexion test is a useful accurate provocative test for cubital tunnel syndrome. ${ }^{14}$

\section{INVESTIGATIONS}

In essence, cubital tunnel syndrome is a clinical diagnosis that is confirmed with nerve conduction studies.

In mild cases, nerve conduction studies may be normal. Electrodiagnostic tests must, however, be interpreted as part of the overall clinical picture. Nathan et al ${ }^{15}$ compared preoperative and postoperative nerve conduction studies and observed that in some patients, although the objective finding of nerve conduction improved markedly, the patients described little or no improvement in symptoms.

$x$ Rays around the elbow may show osteoarthritis, cubitus valgus or calcification in the medial collateral ligament, and should be taken if there is a history of pre-existing trauma or when the symptoms do not fit in with the clinical examination.

Magnetic resonance imaging ${ }^{16}$ and ultrasound ${ }^{17}$ have been proposed as investigations to confirm cubital tunnel syndrome, and are useful in showing lesions such as ganglions, neuromas or aneurysms of the ulnar artery in Guyon's canal causing compression neuropathy. In clinical practice, however, it is unusual to request these tests.

\section{TREATMENT}

\section{Conservative treatment}

A diagnosis of cubital tunnel syndrome does not in itself necessitate surgery. Some authors have emphasised the importance of patient education.

For example, it is reasonable to suggest that the patient avoids provocative activities, such as protracted periods of elbow flexion.

Padua et al ${ }^{18}$ studied the natural history of 24 patients with cubital tunnel syndrome who declined surgery. They observed that about half of their untreated patients reported improvement in symptoms at follow-up. This subjective improvement was supported by improvements in the nerve conduction velocities around the elbow. Most patients reported changing their arm posture after the diagnosis was made. This Italian study seems to confirm the anecdotal observation that mild cases of cubital tunnel syndrome may resolve spontaneously without surgical treatment.

Dellon et al ${ }^{19}$ investigated 128 patients, of whom 43 had bilateral ulnar nerve compression. All patients were initially treated conservatively, although many required subsequent surgery. A history of elbow injury considerably worsened the outcome, but the results of pretreatment electrodiagnosis was not predictive of the need for surgery.

However, in a cooperative patient with objective neurology, most surgeons would recommend surgical release.

\section{Surgical treatment for cubital tunnel syndrome}

All surgical procedures carry risk (box 2), and there is debate within the profession as to when we should operate on this condition.
In practice, surgical treatment is offered for more severe cases and where conservative management is deemed to have failed.

Surgical release involves incising longitudinally over the cubital tunnel to release the surrounding retinacular fibres. This procedure must be performed with some care, as damage to small branches of the nerve may lead to painful neuroma. Some surgeons simply decompress the nerve in situ whereas others attempt to mobilise the nerve freely and transpose it anteriorly out of the cubital tunnel.

Nathan et $a l^{15}$ investigated 102 cases (74 patients) of cubital tunnel syndrome treated by simple decompression in situ. His study found that women did better than men, and also noted that postoperative weight gain was a bad prognostic sign. Interestingly, those patients who also had a carpal tunnel release did better, with the possible implication that many cases of cubital tunnel syndrome are complicated by undiagnosed carpal tunnel syndrome.

Some surgeons believe that a release should be supplemented by medial epicondylectomy. ${ }^{20}{ }^{21}$ This eliminates the medial epicondyle as a source of compression.

The remaining options involve transposition of the ulnar nerve, in which the surgeon moves the nerve anteriorly. This requires complete freeing of the nerve, and some surgeons consider this unnecessary, as damage to the blood supply (the vasa vasorum) may lead to a secondary ischaemic neuritis.

Such an approach also exposes the nerve to the possibility of secondary compression at the level of the ligament of Struthers or at the intermuscular septum, and most modern surgeons would therefore release these structures in the same procedure. The transposed nerve can then be left in one of three locations: subcutaneous, intramuscular or submuscular.

Fitzgerald $e t$ al $^{22}$ retrospectively investigated 20 patients of military service at an average follow-up of 24 months, and found that after submuscular nerve transposition, 19 of 20 patients had returned to active duties. Objective markers of hand function also improved. One patient developed permanent damage to his medial antebrachial cutaneous nerve. Of the 20 patients, 19 said they would undergo the procedure again.

Nabham et al ${ }^{23}$ compared transposition of the ulnar nerve with simple decompression and found no difference in outcome. This randomised study of 66 patients led the authors to recommend decompression in situ for the ulnar nerve, as more elaborate techniques carry the risk of long-term elbow instability.

Some authors investigated the specific problem of longestablished severe ulnar nerve compression (McGowan grade 3). Matsuzaki et $^{2}{ }^{24}$ studied a series of 15 patients with severe cubital tunnel syndrome which included marked wasting of intrinsic muscles, claw hand deformity and immeasurable (electrically silent) nerve conduction studies. Functional improvement beyond 2 years was observed in this group, although patients $>70$ years of age showed a slower recovery (box 3 ).

In the past decade, various authors have described endoscopic release of the ulnar nerve. ${ }^{25} 26$ Tsai et al ${ }^{25}$ described a series of 76 patients ( 85 elbows) treated by endoscopic release of the tunnel. The authors concluded that endoscopic release is a safe and reliable treatment for the condition, particularly in patients with mild to moderate symptoms.

However, it should be remembered that the proponents of endoscopic release are attempting to replace a well-understood and often-performed open procedure with a technically demanding alternative that requires specialist equipment. Open surgery for this condition is not associated with a long or obvious scar, and the obvious gain of endoscopic release- a smaller incision-will not be enough to convince all surgeons of the technique. 


\section{Revision surgery}

The results of revision surgery are often disappointing in cubital tunnel syndrome. Patients aged $>50$ years do particularly badly. Preoperative electromyelogram evidence of denervation is a bad prognostic index.

\section{CONCLUSION}

Cubital tunnel syndrome is the second most common reason for peripheral nerve entrapment neuropathy in the upper limb. It is more common in certain occupations. Patients who have diabetes and those who have sustained injuries or degenerative change around the elbow are also at increased risk.

Education of patients and orthotics may help to relieve symptoms, but in more severe cases surgical release is effective. There is ongoing debate within the profession as to what constitutes the optimum surgical approach. The procedure is associated with some risk of complications, and persistent cases referred for re-exploration may not respond to surgery.

Competing interests: None.

\section{REFERENCES}

1 O'Driscoll SW, Horii E, Carmichael SW, et al. The cubital tunnel and ulnar neuropathy. J Bone Joint Surg [Br] 1991:73:613-17.

2 Siqueira MG, Martins RS. The controversial arcade of Struthers. Surg Neurol. 2005;64(Suppl 1): S1, 17-20; discussion S1, 20-1.

3 Kanazawa S, Fujioka $\mathrm{H}$, Kanatani T, et al. The relation between cubital tunnel syndrome and the elbow alignment. Kobe J Med Sci 1994;40:155-63.

4 Descatha A, Leclerc A, Chastang JF, et al. Incidence of ulnar nerve entrapment at the elbow in repetitive work. Scand J Work Environ Health 2004;30:234-40.

5 Kakosy T. Tunnel syndromes of the upper extremities in workers using handoperated vibrating tools. Med Lav 1994;85:474-80.

6 Aoki $M$, Kanaya $\mathrm{K}$, Aiki $\mathrm{H}$, et al. Cubital tunnel syndrome in adolescent base ball players: a report of 6 cases with 3 to 5 year follow up. Arthroscopy 2005;21:758.

7 Seror P, Nathan PA. Relative frequency of nerve conduction abnormalities at carpal tunnel and cubital tunnel in France and the United States: importance of silent neuropathies and role of ulnar neuropathy after unsuccessful carpal tunnel syndrome release. Ann Chir Main Memb Super 1993;12:281-5.

8 Rydevik B, Lundborg G, Bagge U. Effects of graded compression on intraneural blood blow. An in vivo study on rabbit tibial nerve. J Hand Surg [Am] $1981 ; 6: 3-12$
9 Dahlin LB, Shyu BC, Danielsen N, et al. Effects of nerve compression or ischaemia on conduction properties of myelinated and non-myelinated nerve fibres. An experimental study in the rabbit common peroneal nerve. Acta Physiol Scand 1989; 136:97-105

10 Baba H, Maezawa Y, Uchida K, et al. Cervical myeloradiculopathy with entrapment neuropathy: a study based on the double crush concept. Spinal Cord 1998;36:399-404.

11 Nemoto K, Matsumoto N, Tazaki K, et al. An experimental study on the double crush hypothesis. J Hand Surg [Am], 1987; 12:552-9.

12 Feldman EL, Stevens MJ, Greene DA. Pathogenesis of diabetic neuropathy. Clin Neurosci 1997;4:365-70.

13 Brown IC, Zinar DM. Traumatic and iatrogenic neurological complications after supracondylar humerus fractures in children. J Pediatr Orthop 1995; 15:440-3.

14 Buehler MJ, Thayer DT. The elbow flexion test. A clinical test for the cubital tunnel syndrome. Clin Orthop Relat Res 1998;233:213-16.

15 Nathan PA, Istvan JA, Meadows KD. Intermediate and long term outcomes following simple decompression of the ulnar nerve at the elbow. Chir Main 2005;24:29-34.

16 Bordalo-Rodrigues M, Rosenberg ZS. MR imaging of entrapment neuropathies at the elbow. Magn Reson Imaging Clin N Am 2004;12:247-63.

17 Martinolic C, Bianchi S, Pugliese F, et al. Sonography of entrapment neuropathies in the upper limb (wrist excluded). J Clin Ultrasound 2004;32:438-50.

18 Padua L, Aprile I, Caliandro P, et al. Natural history of ulnar entrapment at the elbow. Clin Neurophysiol 2002;113:1980-4.

19 Dellon AL, Hament W, Gittelshon A. Nonoperative management of cubital tunnel syndrome: an 8-year prospective study. Neurology 1993:43:1673-7.

20 Popa M, Dupert T. Treatment of cubital tunnel syndrome by frontal partial medial epicondylectomy. A retrospective study of 55 cases. J Hand Surg $[\mathrm{Br}]$ 2004;29:563-7.

21 Hicks D, Toby EB. Ulnar nerve strains at the elbow: the effect of in situ decompression and medial epicondylectomy. J Hand Surg [Am] 2003;27:1026-31

22 Fitzgerald BT, Dao KD, Shin AY. Functional outcomes in young active duty military personnel after submuscular ulnar nerve transposition. J Hand Surg [Am], 2004;29:619-24.

23 Nabham A, Ahlhelm F, Kelm J, et al. Simple decompression or subcut anterior transposition of the ulnar nerve for cubital tunnel syndrome. J Hand Surg $[\mathrm{Br}]$ 2005;30:521-4.

24 Matsuzaki H, Yoshizu T, Maki Y, et al. Long term clinical and neurologic recovery in the hand after surgery for severe cubital tunnel syndrome. J Hand Surg [Am] 2004;29:373-8.

25 Tsai TM, Chen IC, Majd ME, et al. Cubital tunnel release with endoscopic assistance: results of a new technique. J Hand Surg [Am] 1999;24:21-9.

26 Hoffmann $\mathbf{R}$, Siemionow $M$. The endoscopic management of cubital tunnel syndrome. J Hand Surg [Br], 2006;31:23-9. 